\title{
Dicyanphosphid-Reaktionen [1]
}

Dicyanophosphide Reactions [1]

Alfred Schmidpeter* und Günther Burget

Institut für Anorganische Chemie der Universität München, Meiserstraße 1, D-8000 München 2

Herrn Professor Dr. Max Schmidt zum 60. Geburtstag gewidmet

Z. Naturforsch. 40b, 1306-1313 (1985); eingegangen am 26. April 1985

Nucleophilic Displacement at Two-coordinate Phosphorus, Sulfuration and Selenation,

${ }^{77}$ Se NMR Spectra, ${ }^{31} \mathrm{P}$ NMR Spectra

${ }^{31} \mathrm{P}$ (and, as applicably, ${ }^{77} \mathrm{Se}$ ) NMR spectra show $\mathrm{P}(\mathrm{CN})_{2}{ }^{-}$to take up one or two atoms of sulfur or selenium and to react with a variety of anionic phosphorus nucleophiles. Displacement of the first cyanide gives cyanodiphosphides, cyanophosphinidene-phosphates, -phosphinates, -thiophosphinates and -iminophosphinates, respectively. Displacement of the second cyanide gives triphosphates and triphosphinates which are otherwise known as products from the nucleophilic $\mathrm{P}_{4}$ degradation.

Das Anion $\mathrm{P}(\mathrm{CN})_{2}{ }^{-}$läßt sich einerseits im Sinne des Cyanverschiebungssatzes als Pseudohalogenid ansehen [2,3], andererseits in komplexchemischer Betrachtung als Cyanidkomplex des Phosphor(I)Ions [4]. Es sollte damit ein - wenn auch im Vergleich zu anderen Phosphiden schwächeres - Nucleophil darstellen, und es sollte andererseits einer nucleophilen Substitution der Cyanreste zugänglich sein, komplexchemisch gesprochen also einem Austausch der Cyanid-Liganden gegen andere [5].

Beide Reaktionsmöglichkeiten sind bereits durch einige Beispiele belegt, so die Nucleophilie von $\mathrm{P}(\mathrm{CN})_{2}{ }^{-}$durch seine Alkylierung zu Dicyan-alkylphosphanen $[2,6]$, durch seine Halogenierung zu Dicyan-halogenphosphanen und Dicyan-dihalogenphosphiten $[7,8]$ und durch die Bildung von Dicyanphosphido-carbonylmetallaten [6]. Mit $\mathrm{CS}_{2}$ reagiert $\mathrm{P}(\mathrm{CN})_{2}{ }^{-}$(etwa im Gegensatz zu $\mathrm{PPh}_{2}^{-}$[9]) jedoch nicht [10]. Weitere Beispiele, in denen $\mathrm{P}(\mathrm{CN})_{2}{ }^{-}$einfach und sogar zweifach als Donor fungiert, liefert seine Umsetzung mit Schwefel [10] und Selen, über deren ${ }^{31} \mathrm{P}-\mathrm{NMR}$-spektroskopische Untersuchung wir im folgenden berichten.

Ein Beispiel für die zweite Reaktionsmöglichkeit bietet die Umsetzung von $\mathrm{P}(\mathrm{CN})_{2}{ }^{-}$mit Phenyl- und $n$-Butyllithium LiR. Sie führt unter Substitution eines Cyanidrestes zum Phenyl-cyanphosphid, das im Rahmen eines Gleichgewichts, bzw. zum n-Butyl-

\footnotetext{
* Sonderdruckanforderungen an Prof. Dr. A. Schmidpeter. Verlag der Zeitschrift für Naturforschung, D-7400 Tübingen $0340-5087 / 85 / 1000-1306 / \$ 01.00 / 0$
}

cyanphosphid, das vollständig in das Cyclophosphan und Cyanid zerfällt [11]:

$$
\begin{aligned}
& \mathrm{P}(\mathrm{CN})_{2}{ }^{-}+\mathrm{R}^{-} \rightarrow \\
& \mathrm{RPCN}^{-}+\mathrm{CN}^{-} \rightleftarrows 1 / 5(\mathrm{RP})_{5}+2 \mathrm{CN}^{-}
\end{aligned}
$$

Offenbar ist das Substitutionsprodukt $\mathrm{RPCN}^{-}$gegen diese Dissoziation um so stabiler, je besser der Rest $\mathrm{R}$ die phosphidische Ladung mit übernehmen (und damit die Lewis-Acidität des Phosphinidens RP anheben) kann. Mit Silyl- und Stannylresten als R sind sie dementsprechend beständig [12]. Das gilt auch für die Produkte mit einem Phosphino-, Phosphinoyl- und Phosphorylrest als R.

Als Ausgangsmaterial diente bei den spektroskopischen Untersuchungen das [18]Krone-6-Kaliumdicyanphosphid, das durch den Cyanidabbau von weißem Phosphor gut zugänglich geworden ist [13]. Für die Gewinnung des Dicyan-dithiophosphats 2 wurde [18]Krone-6-Natriumdicyanphosphid verwendet [2].

\section{Dicyan-thio- und -selenophosphite und -phosphate}

Mit der doppelt molaren Menge Schwefel reagiert $\mathrm{P}(\mathrm{CN})_{2}{ }^{-}$vollständig zum bereits bekannten [14] Dicyan-dithiophosphat 2 ab [10]. Die Reaktion läuft über das Dicyan-thiophosphit $\mathbf{1}$ als Zwischenstufe. $\mathbf{1}$ kann aber nicht einfach durch eine Umsetzung mit der äquimolaren Menge Schwefel erhalten werden. Vielmehr beobachtet man bei diesem Reaktandenverhältnis sowie beim Verhältnis 0,5 und 1,5 jeweils ein Gleichgewichtsgemisch, an dem 1 neben Ausgangs- und Endstufe beteiligt ist:

$$
2 \mathrm{PS}(\mathrm{CN})_{2}{ }^{-} \rightleftarrows \mathrm{P}(\mathrm{CN})_{2}{ }^{-}+\mathrm{PS}_{2}(\mathrm{CN})_{2}{ }^{-}
$$


Die Konstante dieses Disproportionierungsgleichgewichts liegt etwas über dem statistischen Wert 0,25 .

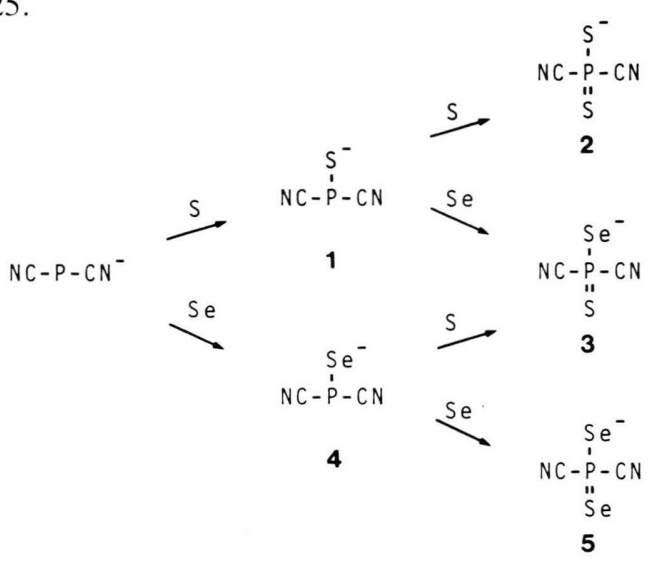

Ein solches Gleichgewichtsgemisch setzt sich mit überschüssigem Selen so um, daß aus 1 das Dicyanthioselenophosphat $\mathbf{3}$ entsteht, während der Anteil an 2 erhalten bleibt und der $\mathrm{P}(\mathrm{CN})_{2}{ }^{-}$-Anteil in 5 (s. u.) übergeht.

Mit überschüssigem Selen reagiert $\mathrm{P}(\mathrm{CN})_{2}{ }^{-}$ausschließlich zum Dicyan-diselenophosphat $\mathbf{5}$, mit weniger als zwei Äquivalenten dagegen wieder zu einem Gleichgewichtsgemisch, das neben $\mathbf{5}$ und $\mathrm{P}(\mathrm{CN})_{2}{ }^{-}$das Dicyan-selenophosphit 4 enthält:

$$
2 \mathrm{PSe}(\mathrm{CN})_{2}{ }^{-} \rightleftarrows \mathrm{P}(\mathrm{CN})_{2}{ }^{-}+\mathrm{PSe}_{2}(\mathrm{CN})_{2}{ }^{-}
$$

Das Gleichgewicht liegt stärker auf der linken Seite als das der Schwefelungsprodukte (Gleichgewichtskonstante etwa 0,1). Ein Gleichgewichtsgemisch mit dem P:Se-Verhältnis 1 setzt sich mit überschüssigem Schwefel vollständig zum Thioselenophosphat 3 um; Schwefel reagiert also offenbar bevorzugt mit 4 ab. Das Selenophosphit 4 wird außerdem von molekularem Sauerstoff rasch oxidiert, so $\mathrm{da} ß$ bei unvollständigem Luftausschlu $\beta$ in den ${ }^{31} \mathrm{P}$ und ${ }^{77}$ Se-NMR-Spektren des Gleichgewichtsgemisches zusätzlich das Signal des Dicyan-selenophosphats 6 auftritt (Tab. I). Durch Zugabe von Sauerstoff kann 4 vollständig in 6 übergeführt werden.

$$
\underset{4}{\mathrm{PSe}(\mathrm{CN})_{2}{ }^{-}+1 / 2 \mathrm{O}_{2}} \rightarrow \underset{\mathbf{6}}{\mathrm{POSe}(\mathrm{CN})_{2}{ }^{-}}
$$

Die NMR-Spektren (Tab. I) belegen die Produkte 1-6 anhand von $\delta^{31} \mathrm{P}, \delta^{77} \mathrm{Se},{ }^{1} \mathrm{~J}_{\mathrm{PSe}}$ und der Satellitenintensität des ${ }^{31} \mathrm{P}$-Signals. Der $\mathrm{S} / \mathrm{Se}$-Austausch führt regelmäßig zu einer Hochfeldverschiebung des Phosphorsignals um 35 bis 50 . Für die Verbindung des
Tab. I. ${ }^{31} \mathrm{P}\left\{{ }^{1} \mathrm{H}\right\}$ - und ${ }^{77} \mathrm{Se}\left\{{ }^{1} \mathrm{H}\right\}$-NMR-Daten (in THF) der Dicyanphosphite $\mathrm{PY}(\mathrm{CN})_{2}{ }^{-}$und Dicyanphosphate $\mathrm{PY}_{2}(\mathrm{CN})_{2}{ }^{-}$.

\begin{tabular}{lllllll}
\hline & $\mathrm{Y}^{1}$ & $\mathrm{Y}^{2}$ & $\delta^{31} \mathrm{P}$ & $\mathrm{I}^{\mathrm{a}}$ & $\delta^{77} \mathrm{Se}^{\mathrm{b}}$ & ${ }^{1} J_{\mathrm{PSe}}[\mathrm{Hz}]$ \\
\hline $\mathbf{1}$ & $\mathrm{S}$ & & $+8,0$ & & & \\
$\mathbf{4}$ & $\mathrm{Se}$ & & $-42,3$ & 0,04 & $-239,7$ & 530 \\
$\mathbf{2}$ & $\mathrm{S}$ & $\mathrm{S}$ & $-8,0$ & & & \\
$\mathbf{6}$ & $\mathrm{O}$ & $\mathrm{Se}$ & $-39,8$ & 0,04 & $+71,7$ & 814 \\
$\mathbf{3}$ & $\mathrm{S}$ & $\mathrm{Se}$ & $-42,8$ & 0,04 & $+158,6$ & 781 \\
$\mathbf{5}$ & $\mathrm{Se}$ & $\mathrm{Se}$ & $-84,8$ & 0,08 & $+171,5$ & 774
\end{tabular}

${ }^{\text {a }}$ Intensität eines ${ }^{77} \mathrm{Se}$-Satelliten relativ zum ${ }^{31} \mathrm{P}$-Hauptsignal;

b bezogen auf $\mathrm{Me}_{2} \mathrm{Se}$.

tetrakoordinierten Phosphors liegt ${ }^{1} J_{\mathrm{PSe}}$ im üblichen Bereich um $800 \mathrm{~Hz}$ und nimmt mit steigender Elektronegativität des zweiten Chalkogens zu. Für das Phosphit 4 ist die Kopplung dagegen deutlich niedriger, wie das aufgrund des geringeren s-Anteils der PSe-Bindung zu erwarten ist. Aus der relativen Intensität der ${ }^{77} \mathrm{Se}$-Satelliten läßt sich die Zahl der an einen Phosphor gebundenen Selenatome ablesen (berechnet aufgrund der natürlichen ${ }^{77} \mathrm{Se}$-Häufigkeit von 7,58\%: 0,041 für ein, 0,082 für zwei Selenatome).

\section{Cyanidphosphide und -diphosphane}

Diphenylphosphid 7 substituiert in $\mathrm{P}(\mathrm{CN})_{2}{ }^{-}$nach Maßgabe des Reaktandenverhältnisses augenblicklich einen oder beide Cyanreste. Es entstehen das 1-Cyan-2,2-diphenyldiphosphid 8 bzw. das 1,1,3,3Tetraphenyltriphosphid 9 (Tab. II). 9 wurde auch bei der Einwirkung von $\mathbf{7}$ auf weißen Phosphor erhalten [15].
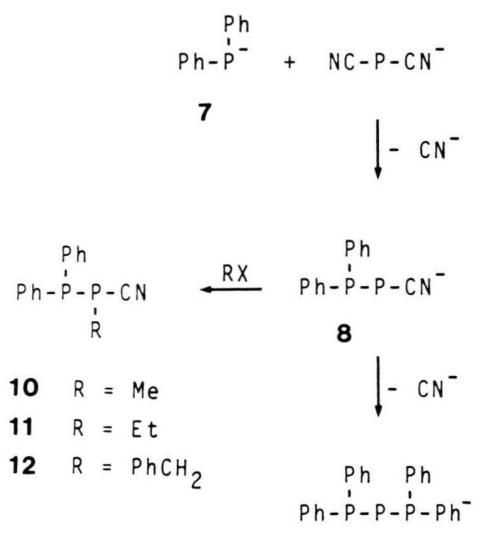

9 
8 reagiert (wie $\mathrm{P}(\mathrm{CN})_{2}{ }^{-}[2,6]$ und $\mathrm{PhPCN}^{-}[11]$ ) mit Alkylhalogeniden ausschließlich am Phosphidzentrum zu den 1-Cyan-1-alkyl-2,2-diphenyldiphosphanen 10-12 (Tab. II). Zum größeren Teil unterliegt $\mathbf{8}$ dabei allerdings einem Redoxzerfall, wobei aus dem als $\mathrm{P}(\mathrm{CN})_{2}{ }^{-}$eingebrachten Phosphor ein Cyclophosphan, aus dem als Diphenylphosphid $\mathbf{7}$ eingebrachten Anteil Cyan-diphenylphosphan, untergeordnet auch Tetraphenyldiphosphan, entsteht [16]:

$$
\mathrm{Ph}_{2} \mathrm{PPCN}^{-}+\mathrm{RX} \rightarrow \frac{1}{n}(\mathrm{RP})_{n}+\mathrm{Ph}_{2} \mathrm{PCN}+\mathrm{X}^{-}
$$

Dieses Ergebnis weist darauf hin, daß die Substitution von $\mathrm{P}(\mathrm{CN})_{2}{ }^{-}$mit 7 zu 8 eine gewisse latente Reduktion beinhaltet, die in der Weiterreaktion z. T. manifest wird. Noch deutlicher wird das bei der oben erwähnten Substitution von $\mathrm{P}(\mathrm{CN})_{2}{ }^{-}$mit Stannid [12].

\section{Cyanphosphinidenphosphinate und -phosphate}

Auch mit Diphenylphosphinit 13 und den Phosphiten $\mathbf{1 4}$ und $\mathbf{1 5}$ setzt sich $\mathrm{P}(\mathrm{CN})_{2}{ }^{-}$momentan je nach Reaktandenverhältnis ein- oder zweistufig um [4].

$$
\begin{aligned}
& \mathrm{Ph} \text { OEt } \mathrm{NMe}_{2} \quad \mathrm{R} \\
& \begin{array}{lll}
13 & 14 & \begin{array}{l}
1 \\
\mathrm{P}-\mathrm{O}^{-} \\
\mathrm{R}
\end{array}
\end{array} \\
& \mid-\mathrm{CN}^{-}
\end{aligned}
$$

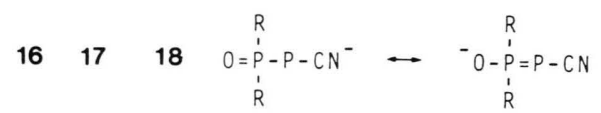

$$
\begin{aligned}
& 13,14,15 \mid-\mathrm{CN}^{-} \\
& 19 \quad 20 \quad 21
\end{aligned}
$$

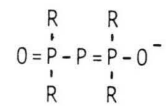

Die Endstufen 19, 20, 21 sind aus den Umsetzungen von 13, 14, 15 mit weißem Phosphor bekannt [19]. Die Produkte der einfachen Substitution 16, 17, 18 können je nach Grenzformel als Phosphinoylbzw. Phosphoryl-cyanphosphid oder als Cyanphosphinidenphosphinat bzw. -phosphat angesprochen werden. Insbesondere bei der Substitution mit $\mathbf{1 4}$ und $\mathbf{1 5}$ ändert sich die chemische Verschiebung des zweifachkoordinierten Phosphors (Tab. II) kaum, was auf die mit $\mathrm{P}(\mathrm{CN})_{2}{ }^{-}$vergleichbare Bindungssi- tuation hinweist. Während sich das Signal des tetrakoordinierten Phosphors $\delta_{\mathrm{B}}$ in der Reihenfolge $\mathrm{R}=$ $\mathrm{Ph}, \mathrm{OEt}, \mathrm{NMe}_{2}$ zu tiefem Feld verschiebt, geht $\delta_{\mathrm{A}}$ in der gleichen Reihenfolge und in etwa dem gleichen $\mathrm{Maß}$ zu hohem Feld.

Während in den voranstehenden Fällen die erste und die zweite Cyanidsubstitution rasch und ohne erkennbare Geschwindigkeitsabstufung ablaufen. wird in anderen Fällen zwar die erste Stufe ebenfalls rasch, die zweite Stufe aber deutlich langsamer oder gar nicht erreicht. Erwartungsgemäß nimmt also der im ersten Schritt anstelle von Cyanid eingeführte Ligand Einfluß auf die Substitutionsgeschwindigkeit.

So beobachtet man mit Diphenylthiophosphinit 22 bei Raumtemperatur sofort nach dem Zusammengeben mit $\mathrm{P}(\mathrm{CN})_{2}{ }^{-}$, aber auch noch nach $3 \mathrm{~d}$ nur das Monosubstitutionsprodukt 23. Bei $60{ }^{\circ} \mathrm{C}$ tritt statt der erwarteten Zweitsubstitution zum Teil eine Entschwefelung des Cyandiphosphiden-thio-phosphinats $\mathbf{2 3}$ zum Cyandiphosphid $\mathbf{8}$ hinzu. Das Thiophosphinit 22 geht dabei in das Dithiophosphinat $\mathrm{Ph}_{2} \mathrm{PS}_{2}$ $\delta^{31} \mathrm{P}=+60,0[20]$ über. 23 ist nun offenbar wieder leichter einer Substitution zugänglich, so daß außerdem das Thiotriphosphinat $\mathbf{2 4}$ entsteht (Tab. II). Daneben beobachtet man noch die Bildung von Tetraphenyldiphosphan.

$$
\begin{aligned}
& 22 \begin{array}{l}
\mathrm{Ph} \\
\mathrm{P}-\mathrm{S}^{-} \\
\mathrm{Ph}
\end{array} \\
& \mid-\mathrm{CN}^{-}
\end{aligned}
$$

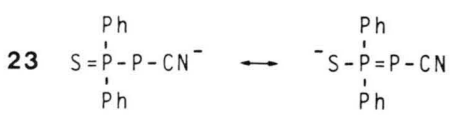

$$
\begin{aligned}
& 22 \mid-P h_{2} P S_{2}- \\
& 8 \quad \begin{array}{l}
P h \\
P h-P-P-C N^{-}
\end{array} \\
& 22 \mid-\mathrm{CN}^{-} \\
& 24 \begin{array}{rl}
P h & P h \\
P h-P-P= & \begin{array}{r}
P \\
P
\end{array}-S^{-} \\
P h
\end{array}
\end{aligned}
$$

Auch mit Diphenylphosphinoanilid 25 bleibt bei Raumtemperatur die Reaktion auf der ersten Substitutionsstufe $\mathbf{2 6}$ stehen, bei $60{ }^{\circ} \mathrm{C}$ wird aber die zweite Substitutionsstufe 27 [21] erreicht (Tab. II). 
Tab. II. ${ }^{31} \mathrm{P}\left\{{ }^{1} \mathrm{H}\right\}$-NMR-Daten (in THF) der Phosphide $\mathrm{XPCN}^{-}(\mathrm{AB}), \mathrm{PX}_{2}^{-}\left(\mathrm{AB}_{2}\right)$ und $\mathrm{X}^{\prime} \mathrm{PX}(\mathrm{ABC})$ und der Biphosphane $\mathrm{Ph}_{2}$ PPRCN $(\mathrm{AB})$. A bezeichnet immer den aus $\mathrm{P}(\mathrm{CN})_{2}{ }^{-}$stammenden Phosphor. $\mathrm{M}^{+}$bezeichnet das als $\mathrm{MX}$ eingebrachte Gegenion neben [18]Krone-6-K $\mathrm{K}^{+}$.

\begin{tabular}{|c|c|c|c|c|c|c|c|c|c|}
\hline $\mathrm{X}$ & $\mathrm{M} / \mathrm{R}$ & & $\delta_{\mathrm{A}}$ & $\delta_{\mathrm{B}}$ & $J_{\mathrm{AB}}[\mathrm{Hz}]$ & & $\delta_{\mathrm{A}}$ & $\delta_{\text {B.C }}$ & $J_{\mathrm{AB}, \mathrm{C}}[\mathrm{Hz}]$ \\
\hline \multirow[t]{4}{*}{$\mathrm{Ph}_{2} \mathrm{P}$} & $\mathrm{K}$ & 8 & $-168,5$ & $+0,3$ & 301,5 & $9[15]$ & $-101,5$ & $+12,2$ & 323,3 \\
\hline & $\mathrm{Me}$ & 10 & $-72,8$ & $-15,1$ & 198,8 & & & & \\
\hline & Et & 11 & $-54,1$ & $-21,0$ & 204,0 & & & & \\
\hline & $\mathrm{PhCH}_{2}$ & 12 & $-55,2$ & $-18,0$ & 212,9 & & & & \\
\hline $\mathrm{Ph}_{2} \mathrm{PO}$ & $\mathrm{Na}$ & 16 & $-161,4$ & $+55,7$ & 362,5 & 19 [19] & $-114,2$ & $+51,7$ & 391,2 \\
\hline$(\mathrm{EtO})_{2} \mathrm{PO}$ & $\mathrm{Na}$ & 17 & $-202,8$ & $+68,5$ & 392,5 & 20 & $-182,9$ & $+67,5$ & 405,3 \\
\hline$\left(\mathrm{Me}_{2} \mathrm{~N}\right)_{2} \mathrm{PO}$ & $\mathrm{Li}$ & 18 & $-205,0$ & $+73,6$ & 347,9 & 21 & $-199,0$ & $+75,4$ & 403,9 \\
\hline $\mathrm{Ph}_{2} \mathrm{PS}$ & $\mathrm{Li}$ & 23 & $-142,0$ & $+54,2$ & 398,2 & & & & \\
\hline $\mathrm{Ph}_{2} \mathrm{P}$ & & & & & & & & $-7,9$ & $280,7^{b}$ \\
\hline $\left.\mathrm{Ph}_{2} \mathrm{PS}\right\}$ & $\mathrm{Li}$ & & & & & 24 & $-91,7$ & $+57,5$ & 481,0 \\
\hline $\mathrm{Ph}_{2} \mathrm{PNPh}$ & $\mathrm{Li}$ & 26 & $-180,0$ & $+22,8$ & 379,5 & $27[21]$ & $-153,3$ & $+32,1$ & 404,7 \\
\hline $\mathrm{PhPN}_{3} \mathrm{MeCPh}$ & $\mathrm{MgBr}$ & 30 & $-178,1$ & $+97,0$ & 404,3 & 31 & $-156,6$ & $+91,9$ & 444,3 \\
\hline $\mathrm{Ph}_{2} \mathrm{PCHPh}$ & $\mathrm{Li}$ & 34 & $-163,0$ & $+57,8$ & 365,7 & & & & \\
\hline $\mathrm{Ph}_{2} \mathrm{PCHPPh}_{2}$ & $\mathrm{Li}$ & $36^{\mathrm{a}}$ & $-174,1$ & $+34,7$ & 353,0 & & & & \\
\hline
\end{tabular}

${ }^{\mathrm{a}} \mathrm{ABC}: \delta_{\mathrm{C}}=-14,7, J_{\mathrm{AC}}=48,3, J_{\mathrm{BC}}=165,2 \mathrm{~Hz} ;{ }^{\mathrm{b}} J_{\mathrm{BC}}=97,5 \mathrm{~Hz}$.

$$
\begin{aligned}
& 25 \begin{array}{l}
\mathrm{Ph} \\
\mathrm{P}-\mathrm{N}-\mathrm{Ph}^{-}+\mathrm{NC}-\mathrm{P}-\mathrm{CN}^{-} \\
\mathrm{Ph}
\end{array} \\
& \mid-\mathrm{CN}^{-} \\
& 26 \quad \begin{array}{c}
P h \\
P h-N=P-P-C N^{-} \\
P h
\end{array} \\
& 25 \mid-\mathrm{CN}^{-}
\end{aligned}
$$

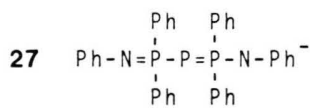

Gleiches gilt für die Umsetzung von $\mathrm{P}(\mathrm{CN})_{2}{ }^{-}$mit dem Anion 29, das aus dem Triazaphosphol 28 [22, 23] mit Phenylmagnesiumbromid erzeugt wird [24].

Die ${ }^{31} \mathrm{P}-\mathrm{NMR}-\mathrm{Spektren}$ (Tab. II) belegen, daß auch 29 in beiden Stufen unter PP-Verknüpfung reagiert [25]; die $\delta^{31} \mathrm{P}-$ Werte von 30 und $\mathbf{3 1}$ schließen sich eng denen von 26 bzw. 27 an, die Kopplungskonstanten ${ }^{1} J_{\mathrm{PP}}$ sind dagegen deutlich größer. Das steht im Einklang mit einem durch den Fünfring bedingten größeren s-Anteil der exocyclischen Bindungen am tetraedrischen Phosphor $\mathrm{P}_{\mathrm{B}}$ und wird in vergleichbarem Maß auch für die PH-Kopplung in Fünfring-Phosphiniminen beobachtet [26]

Während bei den ambidenten Anionen $\mathrm{Ph}_{2} \mathrm{PY}^{-}$ mit $\mathrm{Y}=\mathrm{O}(\mathbf{1 3}), \mathrm{S}(\mathbf{2 2})$ und $\mathrm{NPh}(\mathbf{2 5})$ gegenüber

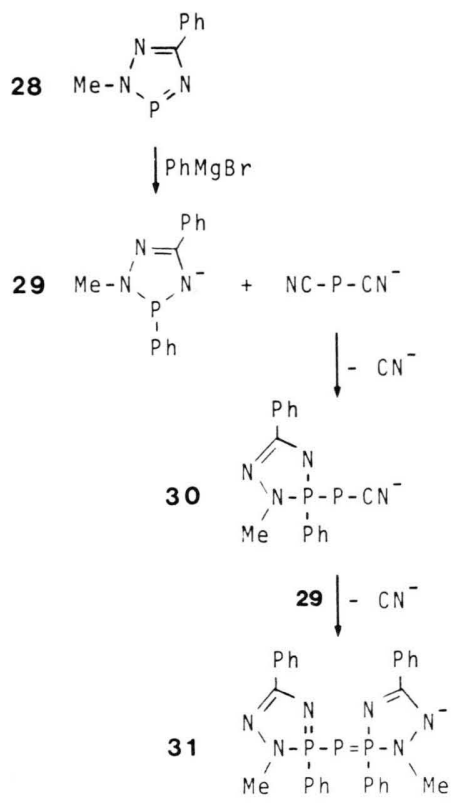

$\mathrm{P}(\mathrm{CN})_{2}{ }^{-}$stets der Phosphor als Nucleophil wirkt, wird ihm bei 32 mit $\mathrm{Y}=\mathrm{CHPh}$ diese Rolle vom Kohlenstoff streitig gemacht. Nur bei tiefer Temperatur $\left(-78^{\circ} \mathrm{C}\right)$ erhält man in der ersten Substitutionsstufe noch das Produkt $\mathbf{3 4}$ mit PP-Verknüpfung (Tab. II). Daneben und bei Raumtemperatur ausschließlich beobachtet man ein zweites Produkt, ${ }^{31} \mathrm{P}\left\{{ }^{1} \mathrm{H}\right\}(\mathrm{THF}): \mathrm{AB}, \delta_{\mathrm{A}}=-97,4, \delta_{\mathrm{B}}=+2,0, J_{\mathrm{AB}}=$ 
$126,8 \mathrm{~Hz}$, das der Substitution durch das Carbanion zuzuordnen ist (33) und das dementsprechend (wie $n$-BuPCN ${ }^{-}$, s.o.) nicht beständig ist, sondern innerhalb weniger Stunden (in nicht geklärter Weise) zerfällt.

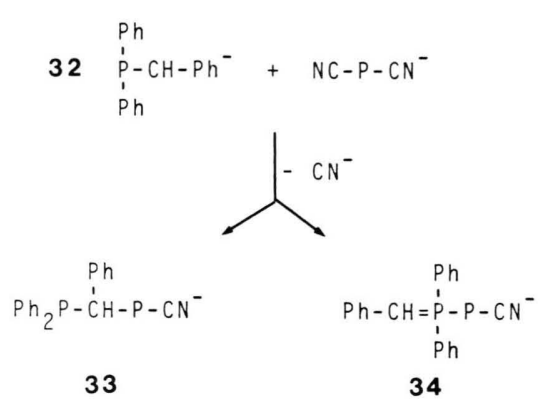

Mit 35 entsteht bei Raumtemperatur unter PPVerknüpfung das Substitutionsprodukt 36, das sich durch sein ${ }^{31} \mathrm{P}-\mathrm{ABC}$-Spinsystem (Tab. II) klar zu erkennen gibt, aber bereits innerhalb von $2 \mathrm{~h}$ wieder vollständig zerfallen ist. Wegen der Unbeständigkeit der Produkte der Erstsubstitution sind mit $\mathbf{3 2}$ und $\mathbf{3 5}$ keine Zweitsubstitutionen möglich [27].

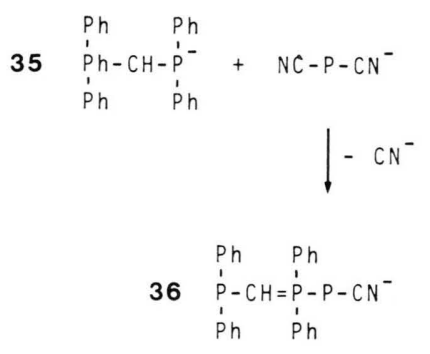

Ganz allgemein und erwartungsgemäß gelingt die Substitution an $\mathrm{P}(\mathrm{CN})_{2}{ }^{-}$nur mit einer im Vergleich zum Cyanid stärkeren Base [5]. Neutrale Phosphane wirken dementsprechend nicht ein [28]. Immerhin erschien uns eine Substitution von Cyanid durch ein Phosphan im zweiten Schritt bei intramolekularer Begünstigung noch möglich. Wir setzten deshalb $\mathrm{P}(\mathrm{CN})_{2}{ }^{-}$bei tiefer Temperatur mit dem zu 32 isomeren $o$-Lithiobenzyl-diphenylphosphan 37 [29] um und erwarteten im ersten Schritt eine der Reaktion mit Phenyllithium [11] entsprechende Substitution zu 38 und erhofften im zweiten Schritt eine intramolekulare Substitution und damit einen Ringschluß zum cyclischen $\lambda^{3}, \lambda^{5}$-Diphosphen $\mathbf{3 9}$.

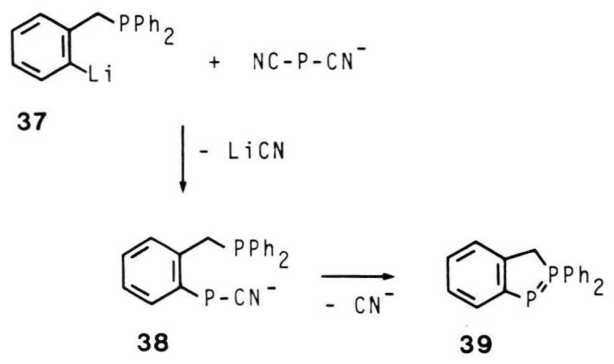

Für beide zeigte das ${ }^{31} \mathrm{P}$-NMR-Spektrum der Reaktionslösung bei $-33{ }^{\circ} \mathrm{C}$ die passenden AB-Signale; für die zweite Stufe belegt insbesondere eine PPKopplung von $407 \mathrm{~Hz}$ die direkte Verknüpfung der beiden Phosphoratome. Dem Erwärmen auf Raumtemperatur halten die Systeme jedoch nicht stand.

Beim zweifachkoordinierten Phosphor sind die $\delta^{31} \mathrm{P}$-Substituentenbeiträge in der Regel angenähert additiv [11]. Das bestätigt sich auch in allen hier neu hinzugekommenen Fällen, in denen die Substitutionsreihe $\mathrm{P}(\mathrm{CN})_{2}{ }^{-}\left(\delta^{31} \mathrm{P}=-193\right), \mathrm{XPCN}^{-}, \mathrm{PX}_{2}{ }^{-}$ (Tab. II) zur Verfügung steht. Das Signal des Mittelglieds liegt dabei stets ein wenig zu höherem Feld als es dem Mittelwert der Endglieder entspricht. Die Kopplungskonstanten ${ }^{1} J_{\mathrm{PP}}$ sind für $\mathrm{XPCN}^{-}$und $\mathrm{X}_{2} \mathrm{P}^{-}$ ähnlich abgestuft und im zweiten Fall stets deutlich größer.

\section{Experimenteller Teil}

\section{Ausgangsverbindungen}

Die Dicyanphosphide [18]Krone-6-NaP(CN $)_{2}$ [2] und $-\mathrm{KP}(\mathrm{CN})_{2}[13]$, sowie die Nucleophile $\mathrm{KPPh}_{2}$ (K-7) [30], $\mathrm{NaOPPh}_{2}(\mathrm{Na}-13)$ [31], $\mathrm{NaOP}(\mathrm{OEt})_{2}$ (Na-14) [10], $\mathrm{LiSPPh}_{2}$ (Li-22) [32], 2-Methyl-3,5diphenyl-2 H-3,4-dihydro-1,2,4,3-triazaphospholylmagnesiumbromid (BrMg-29) [23], $\mathrm{LiCH}\left(\mathrm{PPh}_{2}\right)_{2}$ (Li-35) [33], $\mathrm{LiC}_{6} \mathrm{H}_{4} \mathrm{CH}_{2} \mathrm{PPh}_{2}-2$ (37) [29] wurden in der beschriebenen Weise dargestellt. $\mathrm{LiOP}\left(\mathrm{NMe}_{2}\right)_{2}$ (Li-15) wurde analog $\mathrm{LiSPPh}_{2}[32]$ aus $\mathrm{HPO}\left(\mathrm{NMe}_{2}\right)_{2}$ [34] und $n$-BuLi in THF bei $0{ }^{\circ} \mathrm{C}$ erhalten; ${ }^{31} \mathrm{P}$-NMR: $\delta=+136,2$.

$\mathrm{LiNPhPPh}_{2}$ (Li-25) wurde aus $\mathrm{PhNHPPh}_{2}$ und $n$-BuLi [35] unter Zusatz von TMEDA dargestellt: $\mathrm{Zu} 11,1 \mathrm{~g}(40,0 \mathrm{mmol}) \mathrm{PhNHPPh}_{2}$ in $100 \mathrm{ml}$ Benzol und $7 \mathrm{ml}(46,4 \mathrm{mmol})$ TMEDA werden $28,0 \mathrm{ml}$ einer 1,43 M Lösung (40,0 mmol) $n$-BuLi in $n$-Hexan langsam zugetropft. Die Lösung färbt sich bereits nach den ersten Tropfen gelb und ist nach beendeter Zugabe orange. Für die weitere Umsetzung wird das Lösungsmittel abgezogen und der Rückstand in THF aufgenommen; ${ }^{31}$ P-NMR: $\delta=+36,6$. 
$\mathrm{LiCHPhPPh}_{2}$ (Li-32) wurde nach der für $\mathrm{LiCH}\left(\mathrm{PPh}_{2}\right)_{2}$ angegebenen Vorschrift [33] aus Benzyldiphenylphosphan und $n$-BuLi erhalten; ${ }^{31} \mathrm{P}$ NMR (Benzol, TMEDA): $\delta=-19,1$.

\section{[18]Krone-6-Natrium-dicyanodithiophosphat}

Die Lösung von 3,17 g (8,56 mmol) [18]Krone-6$\mathrm{NaP}(\mathrm{CN})_{2}$ in $20 \mathrm{ml} \mathrm{THF}$ und $0,55 \mathrm{~g}(17,15 \mathrm{mmol})$ Schwefelblüte in $70 \mathrm{ml}$ THF wurden zusammengegeben und bei Raumtemperatur bis zur beginnenden Trübung eingeengt. Über Nacht schieden sich 2,40 g (65\%) farblose Kristalle ab; Schmp. $146-149{ }^{\circ} \mathrm{C}$.

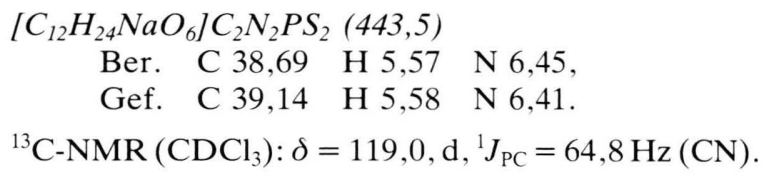

\section{Umsetzung von $\mathrm{P}(\mathrm{CN})_{2}{ }^{-}$mit Schwefel (und Selen)}

Entsprechend den Molverhältnissen 0,5, 1,0 und 1,5 wurden $316 \mathrm{mg} \quad(0,82 \mathrm{mmol}), \quad 291 \mathrm{mg}$ $(0,75 \mathrm{mmol})$ bzw. $251 \mathrm{mg}(0,65 \mathrm{mmol})$ [18]Krone-6$\mathrm{KP}(\mathrm{CN})_{2}$ in $2 \mathrm{ml} \mathrm{THF}$ mit $13 \mathrm{mg}(0,41 \mathrm{mmol})$, $24 \mathrm{mg}(0,75 \mathrm{mmol})$ bzw. $31 \mathrm{mg}(0,97 \mathrm{mmol})$ Schwefel geschüttelt bis dieser gelöst war. Die nach jeweils etwa $4 \mathrm{~h}$ aufgenommenen ${ }^{31} \mathrm{P}-\mathrm{NMR}$-Spektren zeigen die Singuletts von $\mathrm{P}(\mathrm{CN})_{2}{ }^{-}(\delta=-193,3), \mathbf{1}$ und 2 (Tab. I) in der Intensitätsverteilung 55:35:10, 30:40:30 bzw. 10:35:55.

Die Reaktionslösung aus der 1:1-Umsetzung wurde mit überschüssigem (100 mg) Schwefel geschüttelt. Das nach $1 \mathrm{~h}$ aufgenommene ${ }^{31} \mathrm{P}-\mathrm{NMR}-$ Spektrum zeigte ausschließlich das Singulett von 2.

Eine weitere Probe gleicher Art aus $312 \mathrm{mg}$ $(0,81 \mathrm{mmol}) \quad[18] \mathrm{Krone}-6-\mathrm{KP}(\mathrm{CN})_{2}$ und $26 \mathrm{mg}$ $(0,81 \mathrm{mmol})$ Schwefel wurde mit überschüssigem (718 mg) schwarzem Selen geschüttelt. Das ${ }^{31} \mathrm{P}$ NMR-Spektrum zeigte die Signale von 2, 3 und 5 (Tab. I) im Intensitätsverhältnis 30:40:30.

\section{Umsetzung von $\mathrm{P}(\mathrm{CN})_{2}{ }^{-}$mit Selen \\ (und Schwefel bzw. Sauerstoff)}

$318 \mathrm{mg}(0,82 \mathrm{mmol})$ [18] Krone-6-KP(CN $)_{2}$ in $2 \mathrm{ml}$ THF wurden mit einem Überschuß an schwarzem Selen geschüttelt und von der überstehenden Lösung nach 5 min ein ${ }^{31} \mathrm{P}-\mathrm{NMR}$-Spektrum aufgenommen. Es zeigte nur das Signal von 5 (Tab. I).

$405 \mathrm{mg}(1,05 \mathrm{mmol})[18] \mathrm{Krone}-6-\mathrm{KP}(\mathrm{CN})_{2}$ in $2 \mathrm{ml}$ THF wurden mit $83 \mathrm{mg}(1,05 \mathrm{mmol})$ schwarzem Selen versetzt. Die entstandene gelbgrüne Lösung zeigte im ${ }^{31} \mathrm{P}$-Spektrum die Signale von $\mathrm{P}(\mathrm{CN})_{2}{ }^{-}, \mathbf{4}$ und $5 \mathrm{im}$ Verhältnis 20:60:20.

Zur Reaktionslösung wurde ein Überschuß an
Schwefel gegeben, der sich in leicht exothermer Reaktion z. T. löste. Das ${ }^{31}$ P-NMR-Spektrum der überstehenden Lösung zeigte nur das Signal von 3 (Tab. I).

$427 \mathrm{mg}(1,11 \mathrm{mmol})$ [18]Krone-6-KP(CN $)_{2}$ und $131 \mathrm{mg}(1,66 \mathrm{mmol})$ schwarzes Selen wurden in $2 \mathrm{ml}$ THF umgesetzt. Das ${ }^{31} \mathrm{P}-\mathrm{NMR}-\mathrm{Spektrum}$ der Lösung zeigte die Signale von $\mathbf{4}$ und $\mathbf{5}$ in gleicher Intensität.

Die Reaktionslösung wurde mit Sauerstoff durchgeschüttelt. Nach $1 \mathrm{~d}$ hatte sich ein kristalliner Niederschlag von 5 und 6 gebildet. Das ${ }^{31}$ P-NMR-Spektrum der überstehenden Lösung zeigte die Signale von 5 und 6 im Verhältnis 1:3.

\section{Umsetzung von $\mathrm{P}(\mathrm{CN})_{2}{ }^{-}$mit 7 \\ (und Alkylhalogeniden)}

Beim Zugeben von 1,88 mg (4,87 mmol) [18]Krone$6-\mathrm{KP}(\mathrm{CN})_{2}$ zu $18 \mathrm{ml}$ einer roten, 0,27 M THF-Lösung von $\mathrm{KPPh}_{2}(4,87 \mathrm{mmol})$ bildet sich ein feiner farbloser Niederschlag. Das ${ }^{31} \mathrm{P}-\mathrm{NMR}-\mathrm{Spektrum}$ der Lösung zeigte nur das AB-Signal von 8 (Tab. II).

Jeweils $2 \mathrm{ml}$ der Lösung wurden auf $0{ }^{\circ} \mathrm{C}$ gekühlt und $0,034 \mathrm{ml}$ Methyliodid, 0,040 ml Ethylbromid bzw. 0,062 ml Benzylchlorid (d.i. die äquimolare Menge) zugegeben. Dabei verblaßte die orangerote Farbe und es setzte sich ein farbloser Niederschlag ab. Die ${ }^{31}$ P-NMR-Spektren zeigten neue AB-Signale (Tab. II), die den Diphosphanen 10, 11 und 12 zuzuordnen sind, und folgende Singuletts (in Klammern sind die relativen Intensitäten angegeben; für die Diphosphane beziehen sie sich auf den A- oder B-Teil): Neben 10 (10): $\delta=+21,9$ (30), (PMe) $)_{5}$, Vergleichswert +21 [36]; $-34,0$ (30), $\mathrm{Ph}_{2} \mathrm{PCN}$, Vergleichswert -33,8 [37]; -62,9 (30), (PMe) $)_{4}$, Zuordnung in Anlehnung an (PEt) $)_{4}$. Neben 11 (30): $\delta=$ $+11,6(10) ;-15,7(5), \mathrm{Ph}_{4} \mathrm{P}_{2}$, Vergleichswert $-15,2$ [38]; -33,0 (10); -34,0 (30), $\mathrm{Ph}_{2} \mathrm{PCN} ;-67,7$ (15), $(\mathrm{PEt})_{4}$, Vergleichswert $-68,0$ [39]. Neben $12(10): \delta=$ $+11,8$ (5); - 15,7 (5), $\mathrm{Ph}_{4} \mathrm{P}_{2} ;-34,0$ (40), $\mathrm{Ph}_{2} \mathrm{PCN}$; $-56,0(40),\left(\mathrm{PhCH}_{2} \mathrm{P}\right)_{4}$, in Analogie zugeordnet.

\section{Umsetzung von $\mathrm{P}(\mathrm{CN})_{2}{ }^{-}$mit 13, 14, 15}

Je $309 \mathrm{mg}(0,80 \mathrm{mmol})$ [18]Krone-6-KP(CN $)_{2}$ in $1.5 \mathrm{ml} \mathrm{THF}$ und $1.45 \mathrm{ml}$ einer $0,55-\mathrm{M}-\mathrm{NaOPPh}_{2}$-Lösung, $\quad 0,80 \mathrm{ml}$ einer 1,00 $\mathrm{M} \mathrm{NaOP}(\mathrm{OEt})_{2}$-Lösung bzw. $1,38 \mathrm{ml}$ einer $0,58 \mathrm{M} \mathrm{LiOP}\left(\mathrm{NMe}_{2}\right)_{2}$-Lösung $(0,80 \mathrm{mmol})$ in THF wurden zusammengegeben. Dabei schied sich ein feiner Niederschlag ab. Das unmittelbar danach aufgenommene ${ }^{31} \mathrm{P}-\mathrm{NMR}$-Spektrum zeigte nur das AB-Signal von 16, 17 bzw. 18 (Tab. II).

Die Reaktionslösungen wurden auf $1,5 \mathrm{ml}$ eingeengt und die gleichen Mengen noch einmal zugege- 
ben. Das unmittelbar danach aufgenommene ${ }^{31} \mathrm{P}$ NMR-Spektrum zeigte das $\mathrm{A}_{2} \mathrm{~B}$-Signal von 19, 20 bzw. 21 (Tab. II).

\section{Umsetzung von $\mathrm{P}(\mathrm{CN})_{2}{ }^{-}$mit $\mathbf{2 5}$ und $\mathbf{2 9}$}

Die wie vorstehend mit $0,83 \mathrm{ml}$ einer $0,96 \mathrm{M}$ $\mathrm{LiPhNPPh}_{2}$-Lösung bzw. 1,23 $\mathrm{ml}$ einer $0,65 \mathrm{M}$ Lösung von BrMg-29 in THF $(0,80 \mathrm{mmol})$ durchgeführten 1:1-Umsetzungen ergaben sofort die AB-Signale von 26 bzw. 29, die auf Zugabe des zweiten Äquivalents erst nach $24 \mathrm{~h}$ bei $60{ }^{\circ} \mathrm{C}$ vollständig in die ABSignale von $\mathbf{2 7}$ bzw. 31 übergingen.

\section{Umsetzung von $\mathrm{P}(\mathrm{CN})_{2}{ }^{-}$mit 22}

Beim Zusammengeben von $268 \mathrm{mg}(0,69 \mathrm{mmol})$ [18]Krone-6-KP(CN $)_{2}$ in $1 \mathrm{ml}$ THF und von $1,97 \mathrm{ml}$ einer $0,35 \mathrm{M} \mathrm{LiSPPh}_{2}$-Lösung in THF $(0,69 \mathrm{mmol})$ entstand ein feiner farbloser Niederschlag. Das sofort aufgenommene ${ }^{31} \mathrm{P}$-NMR-Spektrum der überstehenden gelben Lösung zeigte das AB-Signal von $\mathbf{2 3}$ (Tab. II) und war nach 3 d unverändert.

Die Lösung wurde auf $1,5 \mathrm{ml}$ eingeengt, nochmals mit dem gleichen Volumen $\mathrm{LiSPPh}_{2}$-Lösung versetzt und $1 \mathrm{~d}$ auf $60{ }^{\circ} \mathrm{C}$ gehalten. Ihr ${ }^{31} \mathrm{P}-\mathrm{NMR}$-Spektrum zeigte danach die $\mathrm{AB}$-Signale von $\mathbf{2 3}$ und $\mathbf{8}$, das ABC-Signal von 24, sowie Singuletts bei $\delta=+60,0$ $\left(\mathrm{Ph}_{2} \mathrm{PS}_{2}^{-}\right),+48,8\left(\mathrm{Ph}_{2} \mathrm{PS}^{-}\right)$und -15,6 $\left(\mathrm{Ph}_{2} \mathrm{PPPh}_{2}\right)$. Ihr Verhältnis (jeweils auf den Phosphor einer bestimmten Umgebung bezogen) betrug etwa $23: 5: 9: 18: 18: 18$.

\section{Umsetzung von $\mathrm{P}(\mathrm{CN})_{2}{ }^{-}$mit 32}

$386 \mathrm{mg}(1,00 \mathrm{mmol})$ [18]Krone-6-KP(CN $)_{2}$ wurden mit $1,54 \mathrm{ml}$ einer $0,65 \mathrm{M} \mathrm{LiPhCH}\left(\mathrm{PPh}_{2}\right)_{2}$-Lösung in THF $(1,00 \mathrm{mmol})$ versetzt. Aus der roten Lösung setzte sich ein farbloser Niederschlag ab. Das nach $10 \mathrm{~min}$ aufgenommene ${ }^{31} \mathrm{P}$-NMR-Spektrum zeigte neben einem schwachen Singulett für unverändertes $\mathbf{3 2}$ das $\mathrm{AB}$-Signal von $\mathbf{3 3}$ mit verhältnismäßig breiten Linien (Tab. II). Seine Intensität war nach $1,5 \mathrm{~h}$ deutlich, nach $1 \mathrm{~d}$ auf Null zurückgegangen. Statt dessen nahm die Intensität eines breiten Multi- pletts bei $\delta=-4$ zu. Dieses kann versuchsweise einem Cyclopentaphosphan (komplexes Multiplett. Verschiebungsbereich +20 bis -10 [40]) zugeordnet werden.

In einem weiteren Versuch wurden die Komponenten beim Zusammengeben auf $-78^{\circ} \mathrm{C}$ gekühlt und rasch danach das ${ }^{31} \mathrm{P}-\mathrm{NMR}$-Spektrum bei $-33^{\circ} \mathrm{C}$ aufgenommen. Es zeigte neben dem AB-Signal von $\mathbf{3 3}$ mit etwa $1 / 10$ Intensität ein zweites von 34, das im Gegensatz zu ersterem auch nach $1 \mathrm{~d}$ noch erhalten war.

\section{Umsetzung von $\mathrm{P}(\mathrm{CN})_{2}{ }^{-}$mit $\mathbf{3 5}$}

$657 \mathrm{mg}(1,70 \mathrm{mmol})$ [18]Krone-6-KP(CN $)_{2}$ und $3,78 \mathrm{ml}$ einer $0,45 \mathrm{M} \mathrm{LiCH}\left(\mathrm{PPh}_{2}\right)_{2}$-Lösung in THF wurden zusammengegeben und davon nach $15 \mathrm{~min}$ ein ${ }^{31} \mathrm{P}-\mathrm{NMR}$-Spektrum aufgenommen. Es zeigt neben einem Singulett bei $\delta=-22,7\left(\mathrm{CH}_{2}\left(\mathrm{PPh}_{2}\right)_{2}\right)$ und einem Multiplett bei $\delta=+2$ (Cyclopentaphosphan?, s. o.) das ABC-Signal von $\mathbf{3 6}$ (Tab. II), das nach $2 \mathrm{~h}$ zugunsten der beiden anderen Signale verschwunden war.

\section{Umsetzung von $\mathrm{P}(\mathrm{CN})_{2}{ }^{-}$mit 37}

$282 \mathrm{mg}(0,73 \mathrm{mmol})$ [18]Krone-6-KP(CN $)_{2}$ wurden mit $260 \mathrm{mg} \mathrm{37} \cdot \mathrm{Et}_{2} \mathrm{O}$ in $2 \mathrm{ml}$ THF geschüttelt und auf $-78^{\circ} \mathrm{C}$ gekühlt. Das bei $-33^{\circ} \mathrm{C}$ aufgenommene ${ }^{31} \mathrm{P}$-NMR-Spektrum zeigte Singuletts (relative Intensität in Klammern) bei $\delta=-10,5(25)$, $\mathrm{Ph}_{2} \mathrm{PCH}_{2} \mathrm{Ph} ;-14,9$ (5), 37; -193,9 (30), $\mathrm{P}(\mathrm{CN})_{2}{ }^{-}$; und AB-Signale bei $\delta_{\mathrm{A}}=+42,3, \delta_{\mathrm{B}}=-113,6$ (je 5), $J_{\mathrm{AB}}=406,4 \mathrm{~Hz}, 39 ; \delta_{\mathrm{A}}=+17,6, \delta_{\mathrm{B}}=-185,4$ (je 25), $J_{\mathrm{AB}}=365,4 \mathrm{~Hz} ; \delta_{\mathrm{A}}=-18,9, \delta_{\mathrm{B}}=-122,0$ (je 10), $J_{\mathrm{AB}}=23,5 \mathrm{~Hz}, 38$. Unmittelbar nach dem Erwärmen auf RT waren keine AB-Signale mehr zu erkennen.

Der Deutschen Forschungsgemeinschaft und dem Fonds der Chemischen Industrie danken wir für die Unterstützung dieser Arbeit, Herrn Konstantin Karaghiosoff für die Aufnahme der ${ }^{77}$ Se-NMR-Spektren. [18]Krone-6-NaPS $(\mathrm{CN})_{2}$ wurde von Herrn Dr. Franz Zwaschka dargestellt.
[1] 11. Mitteilung über Cyanphosphorverbindungen. 10. Mitteilung [6].

[2] A. Schmidpeter und F. Zwaschka, Angew. Chem. 89, 747 (1977); Angew. Chem., Int. Ed. Engl. 16, 704 (1977).

[3] A. Schmidpeter, F. Zwaschka und W. S. Sheldrick, ACS Symposium Series 171, 419 (1981).

[4] A. Schmidpeter, S. Lochschmidt, G. Burget und W. S. Sheldrick, Phosphorus and Sulfur 18, 23 (1983).
[5] Zum Mechanismus der nucleophilen Substitution am zweifachkoordinierten Phosphor vgl. S. Lochschmidt und A. Schmidpeter, Z. Naturforsch. 40b, 765 (1985).

[6] A. Schmidpeter und F. Zwaschka, Z. Chem. 24, 376 (1984).

[7] A. Schmidpeter und F. Zwaschka, Angew. Chem. 91, 441 (1979); Angew. Chem., Int. Ed. Engl. 18, 411 (1979)

[8] W. S. Sheldrick, F. Zwaschka und A. Schmidpeter 
Angew. Chem. 91, 1000 (1979); Angew. Chem., Int. Ed. Engl. 18, 935 (1979).

[9] J. Kopf, R. Lenck, S. N. Olafson und R. Kramolowsky, Angew. Chem. 88, 811 (1976); Angew. Chem., Int. Ed. Engl. 15, 768 (1976).

[10] F. Zwaschka, Dissertation, München 1982.

[11] A. Schmidpeter, K.-H. Zirzow, G. Burget, G. Huttner und I. Jibril, Chem. Ber. 117, 1695 (1984).

[12] A. Schmidpeter und G. Burget, unveröffentlicht.

[13] A. Schmidpeter, G. Burget, F. Zwaschka und W. S. Sheldrick, Z. Anorg. Allg. Chem. 527, 17 (1985).

[14] H. W. Roesky, Chem. Ber. 100, 2138 (1967).

[15] A. Schmidpeter und G. Burget, Phosphorus and Sulfur 22, 323 (1985).

[16] Dabei ist noch zu klären, ob dieser Zerfall über die Diphosphane 10-12 verläuft oder einen unabhängigen Weg nimmt. Von Halogendiphosphanen ist ein disproportionierender Zerfall in Cyclophosphan und Halogenphosphan an einigen Beispielen bekannt $[17,18]$.

[17] H. Vetter und H. Nöth, Chem. Ber. 96, 1816 (1963).

[18] A. Hinke, W. Kuchen und J. Kutter, Angew. Chem. 93, 1112 (1981); Angew. Chem., Int. Ed. Engl. 20, 1060 (1981).

[19] A. Schmidpeter, G. Burget, H. G. von Schnering und D. Weber, Angew. Chem. 96, 795 (1984); Angew. Chem., Int. Ed. Engl. 23, 816 (1984).

[20] Vgl. $\mathrm{Ph}_{2} \mathrm{PS}_{2} \mathrm{H}, \delta^{31} \mathrm{P}=+52,3$ : W. Kuchen und $\mathrm{H}$. Mayatepec, Chem. Ber. 101, 3454 (1968).

[21] A. Schmidpeter und G. Burget, Angew. Chem. 97, 602 (1985); Angew. Chem., Int. Ed. Engl. 24, 580 (1985).

[22] Y. Charbonnel und J. Barrans, Tetrahedron 32, 2039 (1976).

[23] A. Schmidpeter, J. Luber und H. Tautz, Angew. Chem. 89, 554 (1977); Angew. Chem., Int. Ed. Engl. 16, 546 (1977).

[24] M. Haddad, Th. N'Gando M'Pondo, L. Malavaud, L. Lopez und J. Barrans, Phosphorus and Sulfur 20, 333 (1984).
[25] Auch Schwefel reagiert mit dem ambidenten Anion 29 am Phosphor, während dessen Methylierung am 4Stickstoff erfolgt [23].

[26] A. Schmidpeter, H. Roßknecht und K. Schumann, Z. Naturforsch. 25b, 1182 (1970).

[27] Bezüglich der Substitution von $\mathrm{P}(\mathrm{CN})_{2}{ }^{-}$mit Lithiobis(diphenylphosphino)-acetonitril (29, $\mathrm{CN}$ statt $\mathrm{H})$ vgl. [21].

[28] Die auf diesem Weg nicht erhaltenen Substitutionsprodukte $\mathrm{R}_{3} \mathrm{PPCN}$ sind gleichwohl auf einem anderen Weg zugänglich, A. Schmidpeter und S. Lochschmidt, unveröffentlicht.

[29] H.-P. Abicht und K. Issleib, Z. Anorg. Allg. Chem. 447, 53 (1978); Z. Chem. 21, 341 (1981).

[30] K. Issleib und A. Tzschach, Chem. Ber. 92, 1118 (1959).

[31] K. Issleib, B. Walther und E. Fluck, Z. Chem. 8, 67 (1968).

[32] T. Emoto, H. Gomi, M. Yoshifuji, R. Okaraki und N. Inamoto, Bull. Chem. Soc. Jpn. 47, 2449 (1974).

[33] R. Appel, M. Wander und F. Knoll, Chem. Ber. 112, 1093 (1979).

[34] D. Houalla, These, Toulouse 1968, S. 51; A. Zwierzak, Bull. Acad. Polon. Sci. Ser. Sci. Chim. 13, 609 (1965).

[35] J. A. Labinger und J. S. Miller, J. Am. Chem. Soc. 104, 6856 (1982).

[36] W. A. Henderson, Jr., M. Epstein und F. S. Seichter, J. Am. Chem. Soc. 85, 2462 (1963).

[37] I. B. Johns, H. R. DiPietro, R. H. Nealey und J. V. Pustinger, J. Phys. Chem. 70, 924 (1966).

[38] W. Kuchen und H. Buchwald, Chem. Ber. 91, 2871 (1958).

[39] M. Baudler, B. Carlson, W. Böhm und G. Reuschenbach, Z. Naturforsch. 31 b, 558 (1976).

[40] L. R. Smith und J. L. Mills, J. Chem. Soc. Chem. Commun. 1974, 808. 